\title{
EBSD - Probably The Best Measurement In The World??
}

\author{
A. P. Day* \\ * HKL Technology, Majsmarken 1, Hobro, Denmark, DK-9500
}

For a wide range of materials, from "simple" alloys to complex polyphase rocks, electron backscatter diffraction (EBSD) is the perfect tool for automatically measuring local crystallography and relating it to microstructure in the scanning electron microscope [1]. When combined with energy or wavelength dispersive X-ray analysis EBSD also becomes a powerful tool for phase discrimination / identification. Over the last decade, the speed of EBSD analysis has increased from 1 EBSP (electron backscatter pattern) per second to over $100 / \mathrm{s}$ and it is now possible to capture EBSD maps with millions of points in a single overnight run.

This fact alone should give us pause for thought - EBSD is a very powerful technique, but it can't do everything and there is little point in just making pretty maps. For some materials there are still challenges, these will be discussed during the presentation, some are listed below.

1) Specimen preparation - some specimens are hard to prepare and may need careful mechanical / electro-polishing, or even ion-beam milling / FIB-ing. Coating should be avoided if possible.

2) EBSP geometry - it can be difficult to choose the best specimen-phosphor distance and SEM working distance. As the EBSD detector gets closer to the specimen, the EBSPs generally get better and easier to analyse, and the spatial resolution increases with shorter working distance. However, the danger of collision also increases, as can be seen in figure 1.

3) SEM conditions - the correct choice of accelerating voltage, probe current, and the use of "poor" vacuum mode (for insulating materials) has a strong influence on EBSP quality. For primarily historical reasons, most EBSD measurements are done at $20 \mathrm{kV}$ and $70^{\circ}$ tilt. 4) EBSP collection - new, high-speed CCD cameras with binning allow EBSP quality to be balanced against acquisition time and measurement accuracy. In some cases it is essential to map at high speed, particularly for beam sensitive materials - figure 2 shows data from a shell $[2,3]$ mapped at between 70 and 100 EBSPs/second. Extremely fine grained, deformed, or badly prepared specimens can be challenging. EBSP background correction is important.

5) EBSP analysis - good system calibration and the optimisation of band detection / indexing parameters is essential to get high quality EBSD data. It is well worth spending time on this.

6) Pseudosymmetry (see figure 3), crystallographically similar phases (e.g. two fcc phases), and "non-existent" phases (without crystallographic data) also present problems.

7) The final challenge is what to do with all the EBSD data and how to present them in a way that is compact, easy to understand and interesting.

\section{References}

[1] V. Randle, Microtexture Determination and Its Applications, W.S. Maney \& Son / Institute of Materials, Minerals and Mining, ISBN 19-0265-383-1, 2003.

[2] Chateigner, D.; Hedegaard, C. \& Wenk, H.R., Mollusc shell microstructure and crystallography, Journal of Structural Geology, 22, 1723-1735, 2000

[3] For the shell maps, Pat Trimby's skill and perseverance are gratefully acknowledged. 

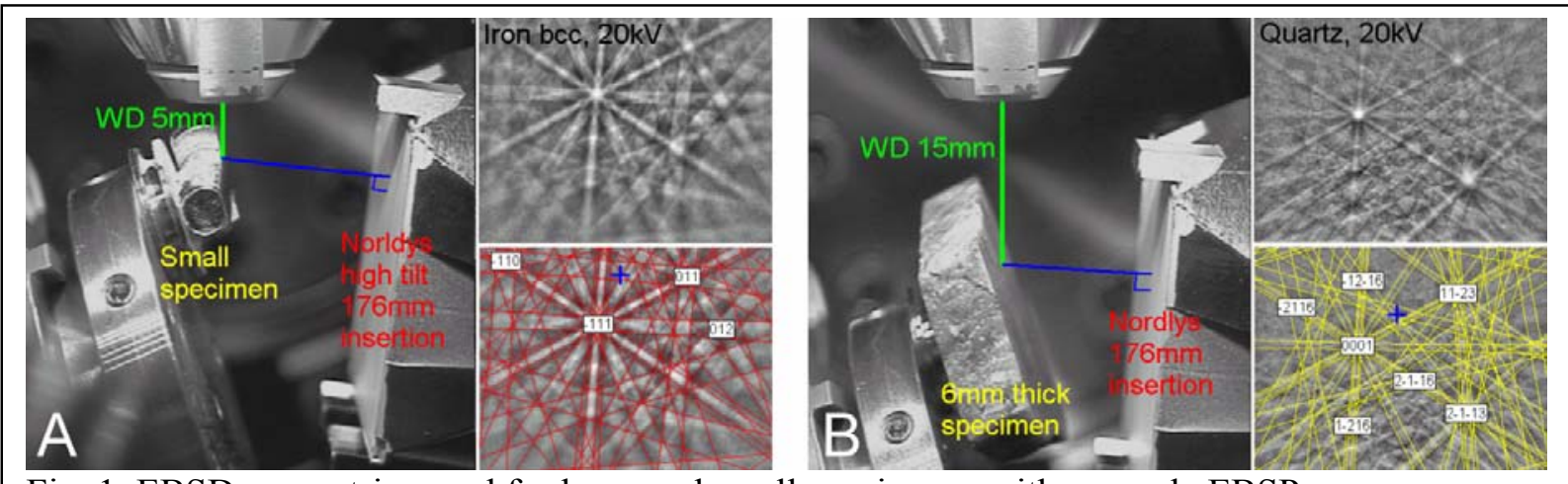

Fig. 1. EBSD geometries used for large and small specimens, with example EBSPs.

A. Small specimen at $5 \mathrm{~mm}$ working distance; B. Larger specimen at $15 \mathrm{~mm}$ working distance.

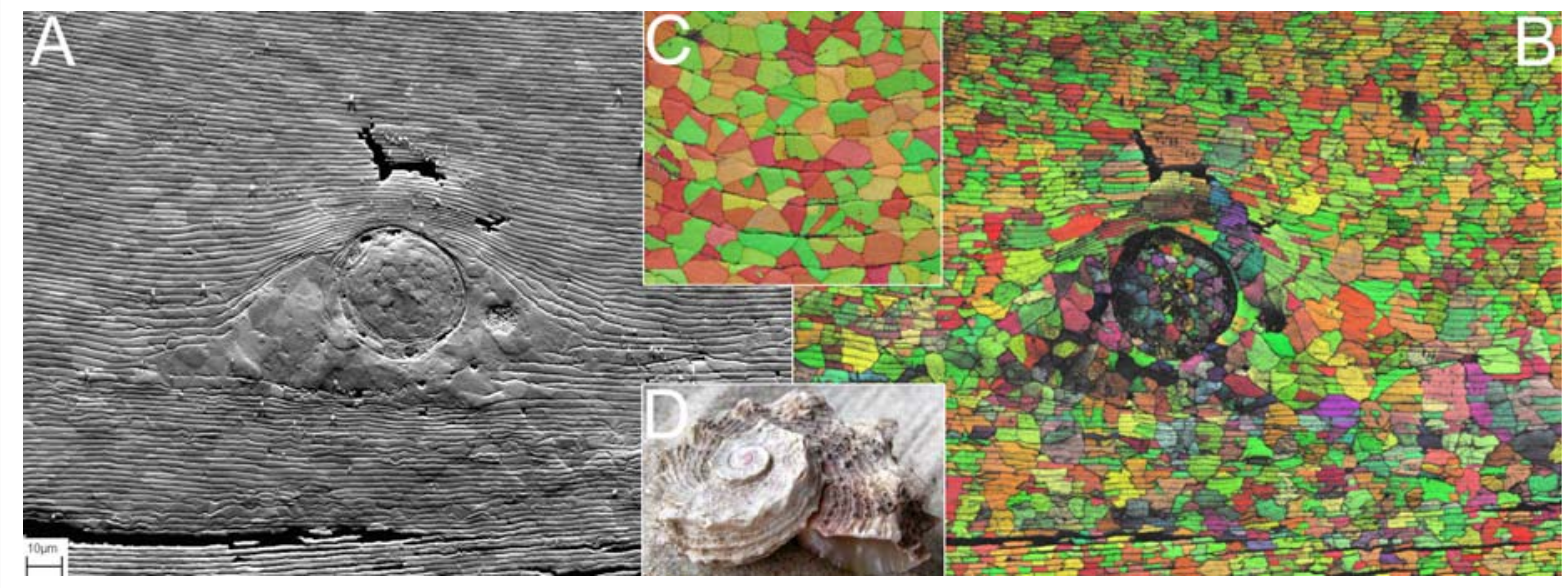

Fig. 2. A. Forescatter image of a "spine" structure in a gastropod shell; B. Orientation map (IPF); C. Orientation map of another area containing wider nacreous layers. D. Angaria delphinus shell
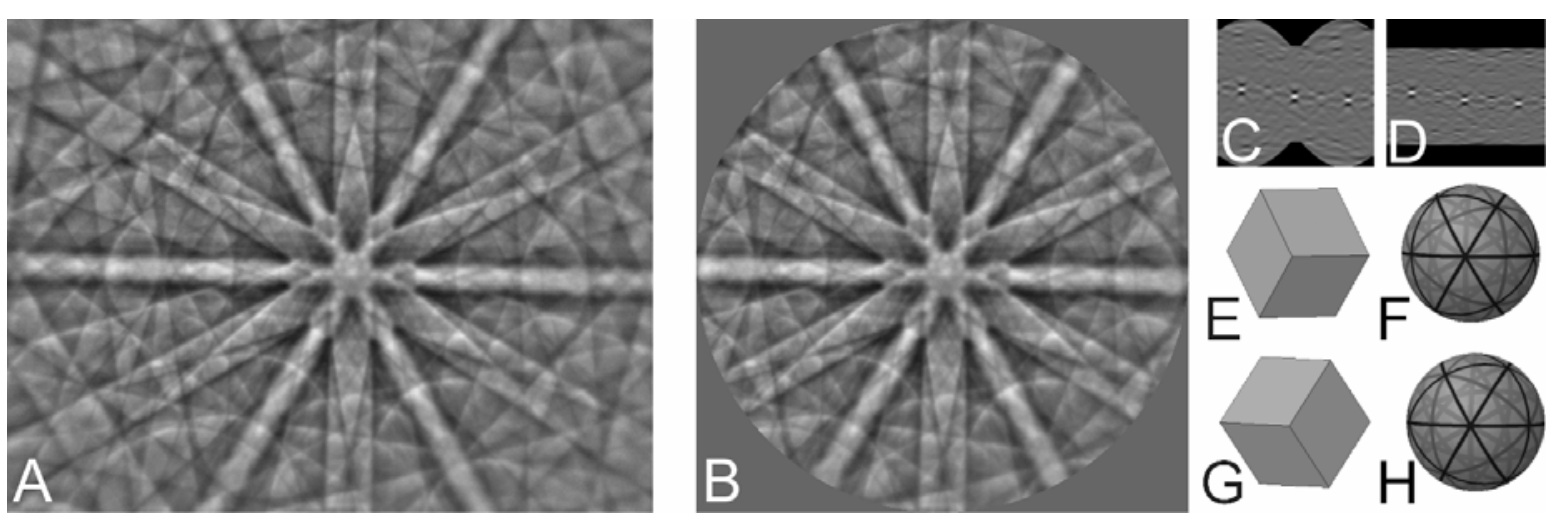

Fig. 3. A. EBSP from bcc iron with $<111>$ near the centre, the weaker Kikuchi bands near the edge of the EBSP indicate which of the two solutions (E or $\mathrm{G}$ ) is correct. B. The EBSP cropped to a circle to show that a larger capture angle is essential for correct indexing; C. \& D. Hough transforms of A \& B; E. \& F. Schematic bcc iron unit cell and spherical Kikuchi map for the correct indexing; G. \& H. As previous, but for the incorrect indexing. 